\title{
Second-order initial value problems with singularities
}

Petio Kelevedjiev ${ }^{1 *}$ and Nedyu Popivanov ${ }^{2}$

Dedicated to Professor Ivan Kiguradze.

"Correspondence:

keleved@mailcity.com

${ }^{1}$ Department of Mathematics,

Technical University of Sofia, Branch

Sliven, Sliven, Bulgaria

Full list of author information is

available at the end of the article

\section{Abstract \\ Using barrier strip arguments, we investigate the existence of $C[0, T] \cap C^{2}(0, T]$-solutions to the initial value problem $x^{\prime \prime}=f\left(t, x, x^{\prime}\right), x(0)=A$, $\lim _{t \rightarrow 0^{+}} x^{\prime}(t)=B$, which may be singular at $x=A$ and $x^{\prime}=B$. \\ MSC: 34B15; 34B16; 34B18}

Keywords: initial value problem; singularities; existence; monotone and positive solutions; barrier strips

\section{Introduction}

In this paper we study the solvability of initial value problems (IVPs) of the form

$$
\begin{aligned}
& x^{\prime \prime}=f\left(t, x, x^{\prime}\right), \\
& x(0)=A, \quad \lim _{t \rightarrow 0^{+}} x^{\prime}(t)=B, \quad B>0 .
\end{aligned}
$$

Here the scalar function $f(t, x, p)$ is defined on a set of the form $\left(D_{t} \times D_{x} \times D_{p}\right) \backslash\left(S_{A} \cup S_{B}\right)$, where $D_{t}, D_{x}, D_{p} \subseteq \mathbb{R}, S_{A}=\mathcal{T}_{1} \times\{A\} \times \mathcal{P}, S_{B}=\mathcal{T}_{2} \times \mathcal{X} \times\{B\}, \mathcal{T}_{i} \subseteq D_{t}, i=1,2, \mathcal{X} \subseteq D_{x}$, $\mathcal{P} \subseteq D_{p}$, and so it may be singular at $x=A$ and $p=B$.

IVPs of the form

$$
\begin{aligned}
& \left(\varphi(t) x^{\prime}(t)\right)^{\prime}=\varphi(t) f(x(t)), \\
& x(0)=A, \quad x^{\prime}(0)=0,
\end{aligned}
$$

have been investigated by Rachůnková and Tomeček [1-3]. For example in [1], the authors have discussed the set of all solutions to this problem with a singularity at $t=0$. Here $A<0, \varphi \in C[0, \infty) \cap C^{1}(0, \infty)$ with $\varphi(0)=0, \varphi^{\prime}(t)>0$ for $t \in(0, \infty)$ and $\lim _{t \rightarrow \infty} \frac{\varphi^{\prime}(t)}{\varphi(t)}=0, f$ is locally Lipschitz on $(-\infty, L]$ with the properties $f(L)=0$ and $x f(x)<0$ for $x \in(-\infty, 0) \cup$ $(0, L)$, where $L>0$ is a suitable constant.

Agarwal and O'Regan [4] have studied the problem

$$
\begin{aligned}
& x^{\prime \prime}=\varphi(t) f\left(t, x, x^{\prime}\right), \quad t \in(0, T], \\
& x(0)=x^{\prime}(0)=0,
\end{aligned}
$$

๑ 2014 Kelevedjiev and Popivanov; licensee Springer. This is an Open Access article distributed under the terms of the Creative Commons Attribution License (http://creativecommons.org/licenses/by/2.0), which permits unrestricted use, distribution, and reproduction in any medium, provided the original work is properly credited. 
where $f(t, x, p)$ may be singular at $x=0$ and/or $p=0$. The obtained results give a positive $C^{1}[0, T] \cap C^{2}(0, T]$-solution under the assumptions that $\varphi \in C[0, T], \varphi(t)>0$ for $t \in(0, T]$, $f:[0, T] \times(0, \infty)^{2} \rightarrow(0, \infty)$ is continuous and

$$
f(t, x, p) \leq[g(x)+h(x)][r(p)+w(p)] \text { for }(t, x, p) \in[0, T] \times(0, \infty)^{2},
$$

where $g, h, r$, and $w$ are suitable functions.

IVPs of the form

$$
\begin{aligned}
& x^{\prime \prime}(t)=f\left(t, x(t), x^{\prime}(t)\right), \quad 0<t<1, \\
& x(0)=x^{\prime}(0)=0,
\end{aligned}
$$

where $f(t, x, p) \in C\left((0,1) \times(0, \infty)^{2}\right)$, maybe singular at $t=0, t=1, x=0$ or $p=0$, have been studied by Yang $[5,6]$. The solvability in $C^{1}[0,1]$ and $C[0,1] \cap C^{2}(0,1)$ is established in these works, respectively, under the assumption that

$$
0<f(t, x, p) \leq k(t) F(x) G(y) \quad \text { for }(t, x, p) \in(0,1) \times(0, \infty)^{2},
$$

where $k, F$, and $G$ are suitable functions.

The solvability of various IVPs has been studied also by Bobisud and O'Regan [7], Bobisud and Lee [8], Cabada and Heikkilä [9], Cabada et al. [10, 11], Cid [12], Maagli and Masmoudi [13], and Zhao [14]. Existence results for problem (1.1), (1.2) with a singularity at the initial value of $x^{\prime}$ have been reported in Kelevedjiev-Popivanov [15].

Here, as usual, we use regularization and sequential techniques. Namely, we proceed as follows. First, by means of the topological transversality theorem [16], we prove an existence result guaranteeing $C^{2}[a, T]$-solutions to the nonsingular IVP for equations of the form (1.1) with boundary conditions

$$
x(a)=A, \quad x^{\prime}(a)=B .
$$

Moreover, we establish the needed $a$ priori bounds by the barrier strips technique. Further, the obtained existence theorem assures $C^{2}[0, T]$-solutions for each nonsingular IVP included in the family

$$
\begin{aligned}
& x^{\prime \prime}=f\left(t, x, x^{\prime}\right), \\
& x(0)=A+n^{-1}, \quad x^{\prime}(0)=B-n^{-1},
\end{aligned}
$$

where $n \in \mathbb{N}$ is suitable. Finally, we apply the Arzela-Ascoli theorem on the sequence $\left\{x_{n}\right\}$ of $C^{2}[0, T]$-solutions thus constructed to (1.3) to extract a uniformly convergent subsequence and show that its limit is a $C[0, T] \cap C^{2}(0, T]$-solution to singular problem (1.1), (1.2). In the case $A \geq 0, B \geq 0$ we establish $C[0, T] \cap C^{2}(0, T]$-solutions with important properties - monotony and positivity.

We have used variants of the approach described above for various boundary value problems (BVPs); see Grammatikopoulos et al. [17], Kelevedjiev and Popivanov [18] and Palamides et al. [19]. For example in [17], we have established the existence of positive 
solutions to the BVP

$$
\begin{aligned}
& g\left(t, x, x^{\prime}, x^{\prime \prime}\right)=0, \quad t \in(0,1), \\
& x(0)=0, \quad x^{\prime}(1)=B, \quad B>0,
\end{aligned}
$$

which may be singular at $x=0$. Note that despite the more general equation of this problem, the conditions imposed here as well as the results obtained are not consequences of those in [17].

\section{Topological transversality theorem}

In this short section we state our main tools - the topological transversality theorem and a theorem giving an important property of the constant maps.

So, let $X$ be a metric space and $Y$ be a convex subset of a Banach space $E$. Let $U \subset Y$ be open in $Y$. The compact map $F: \bar{U} \rightarrow Y$ is called admissible if it is fixed point free on $\partial U$. We denote the set of all such maps by $\mathbf{L}_{\partial u}(\bar{U}, Y)$.

A map $F$ in $\mathbf{L}_{\partial u}(\bar{U}, Y)$ is essential if every map $G$ in $\mathbf{L}_{\partial u}(\bar{U}, Y)$ such that $G|\partial U=F| \partial U$ has a fixed point in $U$. It is clear, in particular, every essential map has a fixed point in $U$.

Theorem 2.1 ([16, Chapter I, Theorem 2.2]) Let $p \in U$ be fixed and $F \in \mathbf{L}_{\partial u}(\bar{U}, Y)$ be the constant map $F(x)=p$ for $x \in \bar{U}$. Then $F$ is essential.

We say that the homotopy $\left\{\mathrm{H}_{\lambda}: X \rightarrow Y\right\}, 0 \leq \lambda \leq 1$, is compact if the map $\mathrm{H}(x, \lambda): X \times$ $[0,1] \rightarrow Y$ given by $\mathrm{H}(x, \lambda) \equiv \mathrm{H}_{\lambda}(x)$ for $(x, \lambda) \in X \times[0,1]$ is compact.

Theorem 2.2 ([16, Chapter I, Theorem 2.6]) Let $Y$ be a convex subset of a Banach space $E$ and $U \subset Y$ be open. Suppose:

(i) $F, G: \bar{U} \rightarrow Y$ are compact maps.

(ii) $G \in \mathbf{L}_{\partial U}(\bar{U}, Y)$ is essential.

(iii) $\mathrm{H}(x, \lambda), \lambda \in[0,1]$, is a compact homotopy joining $F$ and $G$, i.e.

$$
\mathrm{H}(x, 1)=F(x) \text { and } \mathrm{H}(x, 0)=G(x) .
$$

(iv) $\mathrm{H}(x, \lambda), \lambda \in[0,1]$, is fixed point free on $\partial U$.

Then $\mathrm{H}(x, \lambda), \lambda \in[0,1]$, has at least one fixed point in $U$ and in particular there is a $x_{0} \in U$ such that $x_{0}=F\left(x_{0}\right)$.

\section{Nonsingular problem}

Consider the IVP

$$
\left\{\begin{array}{l}
x^{\prime \prime}=f\left(t, x, x^{\prime}\right), \\
x(a)=A, \quad x^{\prime}(a)=B, \quad B \geq 0,
\end{array}\right.
$$

where $f: D_{t} \times D_{x} \times D_{p} \rightarrow \mathbb{R}, D_{t}, D_{x}, D_{p} \subseteq \mathbb{R}$.

We include this problem into the following family of regular IVPs constructed for $\lambda \in$ $[0,1]$

$$
\left\{\begin{array}{l}
x^{\prime \prime}=\lambda f\left(t, x, x^{\prime}\right), \\
x(a)=A, \quad x^{\prime}(a)=B,
\end{array}\right.
$$

and suppose the following. 
(R) There exist constants $T>a, m_{1}, \bar{m}_{1}, M_{1}, \bar{M}_{1}$, and a sufficiently small $\tau>0$ such that

$$
\begin{aligned}
& m_{1} \geq 0, \quad \bar{M}_{1}-\tau \geq M_{1} \geq B \geq m_{1} \geq \bar{m}_{1}+\tau \\
& {[a, T] \subseteq D_{t}, \quad\left[A-\tau, M_{0}+\tau\right] \subseteq D_{x}, \quad\left[\bar{m}_{1}, \bar{M}_{1}\right] \subseteq D_{p}}
\end{aligned}
$$

where $M_{0}=A+M_{1}(T-a)$,

$$
\begin{aligned}
& f(t, x, p) \in C\left([a, T] \times\left[A-\tau, M_{0}+\tau\right] \times\left[m_{1}-\tau, M_{1}+\tau\right]\right), \\
& f(t, x, p) \leq 0 \quad \text { for }(t, x, p) \in[a, T] \times D_{x} \times\left[M_{1}, \bar{M}_{1}\right], \\
& f(t, x, p) \geq 0 \quad \text { for }(t, x, p) \in[a, T] \times D_{M_{0}} \times\left[\bar{m}_{1}, m_{1}\right],
\end{aligned}
$$

where $D_{M_{0}}=D_{x} \cap\left(-\infty, M_{0}\right]$.

Our first result ensures bounds for the eventual $C^{2}$-solutions to (3.2). We need them to prepare the application of the topological transversality theorem.

Lemma 3.1 Let $(\mathrm{R})$ hold. Then each solution $x \in C^{2}[a, T]$ to the family $(3.2)_{\lambda}, \lambda \in[0,1]$, satisfies the bounds

$$
A \leq x(t) \leq M_{0}, \quad m_{1} \leq x^{\prime}(t) \leq M_{1}, \quad m_{2} \leq x^{\prime \prime}(t) \leq M_{2} \quad \text { for } t \in[a, T]
$$

where

$$
\begin{aligned}
& m_{2}=\min \left\{f(t, x, p):(t, x, p) \in[a, T] \times\left[A, M_{0}\right] \times\left[m_{1}, M_{1}\right]\right\} \\
& M_{2}=\max \left\{f(t, x, p):(t, x, p) \in[a, T] \times\left[A, M_{0}\right] \times\left[m_{1}, M_{1}\right]\right\} .
\end{aligned}
$$

Proof Suppose that the set

$$
S_{-}=\left\{t \in[a, T]: M_{1}<x^{\prime}(t) \leq \bar{M}_{1}\right\}
$$

is not empty. Then

$$
x^{\prime}(a)=B \leq M_{1} \quad \text { and } \quad x^{\prime} \in C[a, T]
$$

imply that there exists an interval $[\alpha, \beta] \subset S_{-}$such that

$$
x^{\prime}(\alpha)<x^{\prime}(\beta) .
$$

This inequality and the continuity of $x^{\prime}(t)$ guarantee the existence of some $\gamma \in[\alpha, \beta]$ for which

$$
x^{\prime \prime}(\gamma)>0
$$

Since $x(t), t \in[a, T]$, is a solution of the differential equation, we have $\left(t, x(t), x^{\prime}(t)\right) \in$ $[a, T] \times D_{x} \times D_{p}$. In particular for $\gamma$ we have

$$
\left(\gamma, x(\gamma), x^{\prime}(\gamma)\right) \in S_{-} \times D_{x} \times\left(M_{1}, \bar{M}_{1}\right]
$$


Thus, we apply (R) to conclude that

$$
x^{\prime \prime}(\gamma)=\lambda f\left(\gamma, x(\gamma), x^{\prime}(\gamma)\right) \leq 0
$$

which contradicts the inequality $x^{\prime \prime}(\gamma)>0$. This has been established above. Thus, $S_{-}$is empty and as a result

$$
x^{\prime}(t) \leq M_{1} \quad \text { for } t \in[a, T] .
$$

Now, by the mean value theorem for each $t \in(a, T]$ there exists a $\xi \in(a, t)$ such that

$$
x(t)-x(a)=x^{\prime}(\xi)(t-a)
$$

which yields

$$
x(t) \leq M_{0} \quad \text { for } t \in[a, T] .
$$

This allows us to use (3.3) to show similarly to above that the set

$$
S_{+}=\left\{t \in[a, T]: \bar{m}_{1} \leq x^{\prime}(t)<m_{1}\right\}
$$

is empty. Hence,

$$
0 \leq m_{1} \leq x^{\prime}(t) \text { for } t \in[a, T]
$$

and so

$$
A \leq x(t) \quad \text { for } t \in[a, T] .
$$

To estimate $x^{\prime \prime}(t)$, we observe firstly that $(\mathrm{R})$ implies in particular

$$
f\left(t, x, M_{1}\right) \leq 0 \quad \text { for }(t, x) \in[a, T] \times\left[A, M_{0}\right]
$$

and

$$
f\left(t, x, m_{1}\right) \geq 0 \quad \text { for }(t, x) \in[a, T] \times\left[A, M_{0}\right],
$$

which yield $m_{2} \leq 0$ and $M_{2} \geq 0$. Multiplying both sides of the inequality $\lambda \leq 1$ by $m_{2}$ and $M_{2}$, we get, respectively, $m_{2} \leq \lambda m_{2}$ and $\lambda M_{2} \leq M_{2}$. On the other hand, we have established

$$
x(t) \in\left[A, M_{0}\right] \quad \text { and } \quad x^{\prime}(t) \in\left[m_{1}, M_{1}\right] \quad \text { for } t \in[a, T] .
$$

Thus,

$$
m_{2} \leq \lambda m_{2} \leq \lambda f\left(t, x(t), x^{\prime}(t)\right) \leq \lambda M_{2} \leq M_{2} \quad \text { for } t \in[a, T]
$$


and each $\lambda \in[0,1]$ and so

$$
x^{\prime \prime}(t) \in\left[m_{2}, M_{2}\right] \quad \text { for } t \in[a, T] .
$$

Let us mention that some analogous results have been obtained in Kelevedjiev [20]. For completeness of our explanations, we present the full proofs here.

Now we prove an existence result guaranteeing the solvability of IVP (3.1).

Theorem 3.2 Let $(\mathrm{R})$ hold. Then nonsingular problem (3.1) has at least one non-decreasing solution in $C^{2}[a, T]$.

Proof Preparing the application of Theorem 2.2, we define first the set

$$
U=\left\{x \in C_{I}^{2}[a, T]: A-\tau<x<M_{0}+\tau, m_{1}-\tau<x^{\prime}<M_{1}+\tau, m_{2}-\tau<x^{\prime \prime}<M_{2}+\tau\right\},
$$

where $C_{I}^{2}[a, T]=\left\{x \in C^{2}[a, T]: x(a)=A, x^{\prime}(a)=B\right\}$. It is important to notice that according to Lemma 3.1 all $C^{2}[a, T]$-solutions to family (3.2) are interior points of $U$. Further, we introduce the continuous maps

$$
\begin{array}{ll}
j: C_{I}^{2}[a, T] \rightarrow C^{1}[a, T] & \text { by } j x=x, \\
V: C_{I}^{2}[a, T] \rightarrow C[a, T] & \text { by } V x=x^{\prime \prime},
\end{array}
$$

and for $t \in[a, T]$ and $x(t) \in j(\bar{U})$ the map

$$
\Phi: C^{1}[a, T] \rightarrow C[a, T] \quad \text { by }(\Phi x)(t)=f\left(t, x(t), x^{\prime}(t)\right) .
$$

Clearly, the map $\Phi$ is also continuous since, by assumption, the function $f\left(t, x(t), x^{\prime}(t)\right)$ is continuous on $[a, T]$ if

$$
x(t) \in\left[m_{0}-\tau, M_{0}+\tau\right] \quad \text { and } \quad x^{\prime}(t) \in\left[m_{1}-\tau, M_{1}+\tau\right] \quad \text { for } t \in[a, T] .
$$

In addition we verify that $V^{-1}$ exists and is also continuous. To this aim we introduce the linear map

$$
W: C_{I_{0}}^{2}[a, T] \rightarrow C[a, T]
$$

defined by $W x=x^{\prime \prime}$, where $C_{I_{0}}^{2}[a, T]=\left\{x \in C^{2}[a, T]: x(a)=0, x^{\prime}(a)=0\right\}$. It is one-to-one because each function $x \in C_{I_{0}}^{2}[a, T]$ has a unique image, and each function $y \in C[a, T]$ has a unique inverse image which is the unique solution to the IVP

$$
x^{\prime \prime}=y, \quad x(a)=0, \quad x^{\prime}(a)=0 .
$$

It is not hard to see that $W$ is bounded and so, by the bounded inverse theorem, the map $W^{-1}$ exists and is linear and bounded. Thus, it is continuous. Now, using $W^{-1}$, we define

$$
V^{-1}: C[a, T] \rightarrow C_{I}^{2}[a, T] \quad \text { by }\left(V^{-1} y\right)(t)=\ell(t)+\left(W^{-1} y\right)(t),
$$


where $\ell(t)=B(t-a)+A$ is the unique solution of the problem

$$
x^{\prime \prime}=0, \quad x(a)=A, \quad x^{\prime}(a)=B .
$$

Clearly, $V^{-1}$ is continuous since $W^{-1}$ is continuous.

We already can introduce a homotopy

$$
\mathrm{H}: \bar{U} \times[0,1] \rightarrow C_{I}^{2}[a, T]
$$

defined by

$$
\mathrm{H}(x, \lambda) \equiv \mathrm{H}_{\lambda}(x) \equiv \lambda V^{-1} \Phi j(x)+(1-\lambda) \ell .
$$

It is well known that $j$ is completely continuous, that is, $j$ maps each bounded subset of $C_{I}^{2}[a, T]$ into a compact subset of $C^{1}[a, T]$. Thus, the image $j(\bar{U})$ of the bounded set $U$ is compact. Now, from the continuity of $\Phi$ and $V^{-1}$ it follows that the sets $\Phi(j(\bar{U}))$ and $V^{-1}(\Phi(j(\bar{U})))$ are also compact. In summary, we have established that the homotopy is compact. On the other hand, for its fixed points we have

$$
\lambda V^{-1} \Phi j(x)+(1-\lambda) \ell=x
$$

and

$$
V x=\lambda \Phi j(x)
$$

which is the operator form of family (3.2). So, each fixed point of $\mathrm{H}_{\lambda}$ is a solution to (3.2), which, according to Lemma 3.1, lies in $U$. Consequently, the homotopy is fixed point free on $\partial U$.

Finally, $\mathrm{H}_{0}(x)$ is a constant map mapping each function $x \in \bar{U}$ to $\ell(t)$. Thus, according to Theorem 2.1, $\mathrm{H}_{0}(x)=\ell$ is essential.

So, all assumptions of Theorem 2.2 are fulfilled. Hence $\mathrm{H}_{1}(x)$ has a fixed point in $U$ which means that the IVP of (3.2) obtained for $\lambda=1$ (i.e. (3.1)) has at least one solution $x(t)$ in $C^{2}[a, T]$. From Lemma 3.1 we know that

$$
x^{\prime}(t) \geq m_{1} \geq 0 \quad \text { for } t \in[a, T],
$$

from which its monotony follows.

The validity of the following results follows similarly.

Theorem 3.3 Let $B>0$ and let $(\mathrm{R})$ hold for $m_{1}>0$. Then problem (3.1) has at least one strictly increasing solution in $C^{2}[a, T]$.

Theorem 3.4 Let $A>0(A=0)$ and let $(\mathrm{R})$ hold for $m_{1}=0$. Then problem (3.1) has at least one positive (nonnegative) non-decreasing solution in $C^{2}[a, T]$.

Theorem 3.5 Let $A \geq 0, B>0$ and let $(\mathrm{R})$ hold for $m_{1}>0$. Then problem (3.1) has at least one strictly increasing solution in $C^{2}[a, T]$ with positive values for $t \in(a, T]$. 


\section{A problem singular at $x$ and $x^{\prime}$}

In this section we study the solvability of singular IVP (1.1), (1.2) under the following assumptions.

$\left(\mathrm{S}_{1}\right)$ There are constants $T>0, m_{1}, \bar{m}_{1}$ and a sufficiently small $v>0$ such that

$$
\begin{aligned}
& m_{1}>0, \quad B>m_{1} \geq \bar{m}_{1}+v, \\
& {[0, T] \subseteq D_{t}, \quad\left(A, \tilde{M}_{0}+v\right] \subseteq D_{x}, \quad\left[\bar{m}_{1}, B\right) \subseteq D_{p},}
\end{aligned}
$$

where $\tilde{M}_{0}=A+B T+1$,

$$
\begin{aligned}
& f(t, x, p) \in C\left([0, T] \times\left(A, \tilde{M}_{0}+v\right] \times\left[m_{1}-v, B\right)\right), \\
& f(t, x, p) \leq 0 \quad \text { for }(t, x, p) \in\left([0, T] \times D_{x} \times\left[m_{1}, B\right)\right) \backslash S_{A}
\end{aligned}
$$

and

$$
f(t, x, p) \geq 0 \quad \text { for }(t, x, p) \in\left([0, T] \times D_{\tilde{M}_{0}} \times\left[\bar{m}_{1}, m_{1}\right]\right) \backslash S_{A},
$$

where $D_{\tilde{M}_{0}}=\left(-\infty, \tilde{M}_{0}\right] \cap D_{x}$.

$\left(\mathrm{S}_{2}\right)$ For some $\alpha \in(0, T]$ and $\mu \in\left(m_{1}, B\right)$ there exists a constant $k<0$ such that $k \alpha+B>\mu$ and

$$
f(t, x, p) \leq k<0 \quad \text { for }(t, x, p) \in[0, \alpha] \times\left(A, \tilde{M}_{0}\right] \times[\mu, B),
$$

where $T, m_{1}$ and $\tilde{M}_{0}$ are as in $\left(\mathrm{S}_{1}\right)$.

Now, for $n \geq n_{\alpha, \mu}$, where $n_{\alpha, \mu}>\max \left\{\alpha^{-1},(B+k \alpha-\mu)^{-1}\right\}$, and $\alpha, \mu$, and $k$ are as in $\left(\mathrm{S}_{2}\right)$, we construct the following family of regular IVPs:

$$
\left\{\begin{array}{l}
x^{\prime \prime}=f\left(t, x, x^{\prime}\right), \\
x(0)=A+n^{-1}, \quad x^{\prime}(0)=B-n^{-1} .
\end{array}\right.
$$

Notice, for $n \geq n_{\alpha, \mu}$, that we have $B-n^{-1}>\mu-k \alpha>\mu>m_{1}>0$.

Lemma 4.1 Let $\left(\mathrm{S}_{1}\right)$ and $\left(\mathrm{S}_{2}\right)$ hold and let $x_{n} \in C^{2}[0, T], n \geq n_{\alpha, \mu}$, be a solution to (4.3) such that

$$
A<x_{n}(t) \leq \tilde{M}_{0} \quad \text { and } \quad m_{1} \leq x_{n}^{\prime}(t)<B \quad \text { for } t \in[0, T] .
$$

Then the following bound is satisfied for each $n \geq n_{\alpha, \mu}$ :

$$
x_{n}^{\prime}(t)<\phi_{\alpha}(t)<B \quad \text { for } t \in(0, T],
$$

where $\phi_{\alpha}(t)= \begin{cases}k t+B, & t \in[0, \alpha], \\ k \alpha+B, & t \in(\alpha, T] .\end{cases}$

Proof Since for each $n \geq n_{\alpha, \mu}$ we have

$$
x_{n}^{\prime}(0)=B-n^{-1}>\mu-k \alpha>\mu,
$$


we will consider the proof for an arbitrary fixed $n \geq n_{\alpha, \mu}$, considering two cases. Namely, $x_{n}^{\prime}(t)>\mu$ for $t \in[0, \alpha]$ is the first case and the second one is $x_{n}^{\prime}(t)>\mu$ for $t \in[0, \beta)$ with $x_{n}^{\prime}(\beta)=\mu$ for some $\beta \in(0, \alpha]$.

Case 1. From $\mu<x_{n}^{\prime}(t) \leq B, t \in[0, \alpha]$, and $\left(\mathrm{S}_{2}\right)$ we have

$$
x_{n}^{\prime \prime}(t)=f\left(t, x_{n}(t), x_{n}^{\prime}(t)\right) \leq k \quad \text { for } t \in[0, \alpha],
$$

i.e. $x_{n}^{\prime \prime}(t) \leq k$ for $t \in[0, \alpha]$. Integrating the last inequality from 0 to $t$ we get

$$
x_{n}^{\prime}(t)-x_{n}^{\prime}(0) \leq k t, \quad t \in[0, \alpha]
$$

which yields

$$
x_{n}^{\prime}(t) \leq k t+B-n^{-1} \quad \text { for } t \in[0, \alpha] .
$$

Now $m_{1} \leq x_{n}^{\prime}(t)<B, t \in[0, T]$, and (4.2) imply

$$
x_{n}^{\prime \prime}(t)=f\left(t, x_{n}(t), x_{n}^{\prime}(t)\right) \leq 0 \quad \text { for } t \in[0, T] .
$$

In particular $x_{n}^{\prime \prime}(t) \leq 0$ for $t \in[\alpha, T]$, thus

$$
x_{n}^{\prime}(t) \leq x_{n}^{\prime}(\alpha) \leq k \alpha+B-n^{-1} \text { for } t \in(\alpha, T] .
$$

Case 2. As in the first case, we derive

$$
x_{n}^{\prime}(t) \leq k t+B-n^{-1} \quad \text { for } t \in[0, \beta] .
$$

On the other hand, since $m_{1} \leq x_{n}^{\prime}(t)<B$ for $t \in[\beta, T]$, again from (4.2) it follows that

$$
x_{n}^{\prime \prime}(t)=f\left(t, x_{n}(t), x_{n}^{\prime}(t)\right) \leq 0 \quad \text { for } t \in[\beta, T]
$$

which yields

$$
x_{n}^{\prime}(t) \leq x_{n}^{\prime}(\beta)=\mu<k \alpha+B-n^{-1} \leq k t+B-n^{-1} \quad \text { for } t \in[\beta, \alpha]
$$

and

$$
x_{n}^{\prime}(t)<k \alpha+B-n^{-1} \quad \text { for } t \in(\alpha, T] .
$$

So, as a result of the considered cases we get

$$
x_{n}^{\prime}(t) \leq\left\{\begin{array}{ll}
k t+B-n^{-1}, & t \in[0, \alpha], \\
k \alpha+B-n^{-1}, & t \in(\alpha, T]
\end{array}<\phi_{\alpha}(t) \quad \text { for } t \in[0, T] \text { and } n \geq n_{\alpha, \mu},\right.
$$

from which the assertion follows immediately.

Having this lemma, we prove the basic result of this section. 
Theorem 4.2 Let $\left(\mathrm{S}_{1}\right)$ and $\left(\mathrm{S}_{2}\right)$ hold. Then singular IVP (1.1), (1.2) has at least one strictly increasing solution in $C[0, T] \cap C^{2}(0, T]$ such that

$$
m_{1} t+A \leq x(t) \leq B t+A \quad \text { for } t \in[0, T], \quad m_{1} \leq x^{\prime}(t)<B \quad \text { for } t \in(0, T]
$$

Proof For each fixed $n \geq n_{\alpha, \mu}$ introduce $\tau=\min \left\{(2 n)^{-1}, \nu\right\}$,

$$
M_{1}=B-n^{-1}, \quad \bar{M}_{1}=B-(2 n)^{-1} \quad \text { and } \quad M_{0}=\left(B-n^{-1}\right) T+A+1<\tilde{M}_{0}
$$

having the properties

$$
\begin{aligned}
& \bar{M}_{1}-\tau>M_{1}=B-n^{-1}>\mu-k \alpha>\mu>m_{1} \geq \bar{m}_{1}+\tau, \\
& {[0, T] \subseteq D_{t}, \quad\left[A+n^{-1}-\tau, M_{0}+\tau\right] \subseteq\left(A, \tilde{M}_{0}+\tau\right] \subseteq D_{x}}
\end{aligned}
$$

and $\left[\bar{m}_{1}, \bar{M}_{1}\right] \subseteq D_{p}$ since $\bar{M}_{1}=B-(2 n)^{-1}<B$. Besides,

$$
\begin{array}{ll}
f(t, x, p) \leq 0 & \text { for }(t, x, p) \in\left([0, T] \times D_{x} \times\left[M_{1}, \bar{M}_{1}\right]\right) \backslash S_{A}, \\
f(t, x, p) \geq 0 & \text { for }(t, x, p) \in\left([0, T] \times\left(D_{x} \times\left(-\infty, M_{0}\right]\right) \times\left[\bar{m}_{1}, m_{1}\right]\right) \backslash S_{A}
\end{array}
$$

and, in view of (4.1),

$$
f(t, x, p) \in C\left([0, T] \times\left[A+n^{-1}-\tau, M_{0}+v\right] \times\left[m_{1}-\tau, M_{1}+\tau\right]\right) .
$$

All this implies that for each $n \geq n_{\alpha, \mu}$ the corresponding IVP of family (4.3) satisfies (R). Thus, we apply Theorem 3.2 to conclude that (4.3) has a solution $x_{n} \in C^{2}[0, T]$ for each $n \geq n_{\alpha, \mu}$. We can use also Lemma 3.1 to conclude that for each $n \geq n_{\alpha, \mu}$ and $t \in[0, T]$ we have

$$
A<A+n^{-1} \leq x_{n}(t) \leq M_{0}<\tilde{M}_{0}
$$

and

$$
m_{1} \leq x_{n}^{\prime}(t) \leq B-n^{-1}<B
$$

Now, these bounds allow the application of Lemma 4.1 from which one infers that for each $n \geq n_{\alpha, \mu}$ and $t \in[0, T]$ the bounds

$$
m_{1} \leq x_{n}^{\prime}(t)<\phi_{\alpha}(t) \leq B
$$

hold. For later use, integrating the least inequality from 0 to $t, t \in(0, T]$, we get

$$
m_{1} t+A+n^{-1} \leq x_{n}(t)<B t+A+n^{-1} \text { for } t \in[0, T]
$$

and $n \geq n_{\alpha, \mu}$.

We consider firstly the sequence $\left\{x_{n}\right\}$ of $C^{2}[0, T]$-solutions of (4.3) only for each $n \geq n_{\alpha, \mu}$. Clearly, for each $n \geq n_{\alpha, \mu}$ we have in particular

$$
x_{n}^{\prime}(t) \geq m_{1}>0 \quad \text { for } t \in[\alpha, T],
$$


which together with (4.6) gives

$$
x_{n}(t) \geq x_{n}(\alpha) \geq m_{1} \alpha+A+n^{-1}>A_{1}>A \quad \text { for } t \in[\alpha, T] \text {, }
$$

where $A_{1}=m_{1} \alpha+A$. On combining the last inequality and (4.4) we obtain

$$
A_{1}<x_{n}(t)<\tilde{M}_{0} \quad \text { for } t \in[\alpha, T], n \geq n_{\alpha, \mu} .
$$

From (4.5) we have in addition

$$
m_{1} \leq x_{n}^{\prime}(t)<\phi_{\alpha}(\alpha)=B+k \alpha \quad \text { for } t \in[\alpha, T], n \geq n_{\alpha, \mu} .
$$

Now, using the fact that (4.1) implies continuity of $f(t, x, p)$ on the compact set $[\alpha, T] \times$ $\left[A_{1}, \tilde{M}_{0}\right] \times\left[m_{1}, \phi_{\alpha}(\alpha)\right]$ and keeping in mind that for each $n \geq n_{\alpha, \mu}$

$$
x_{n}^{\prime \prime}(t)=f\left(t, x_{n}(t), x_{n}^{\prime}(t)\right) \quad \text { for } t \in[\alpha, T],
$$

we conclude that there is a constant $M_{2}$, independent of $n$, such that

$$
\left|x_{n}^{\prime \prime}(t)\right| \leq M_{2} \quad \text { for } t \in[\alpha, T] \text { and } n \geq n_{\alpha, \mu} \text {. }
$$

Using the obtained a priori bounds for $x_{n}(t), x_{n}^{\prime}(t)$ and $x_{n}^{\prime \prime}(t)$ on the interval $[\alpha, T]$, we apply the Arzela-Ascoli theorem to conclude that there exists a subsequence $\left\{x_{n_{k}}\right\}, k \in \mathbb{N}$, $n_{k} \geq n_{\alpha, \mu}$, of $\left\{x_{n}\right\}$ and a function $x_{\alpha} \in C^{1}[\alpha, T]$ such that

$$
\left\|x_{n_{k}}-x_{\alpha}\right\|_{1} \rightarrow 0 \quad \text { on the interval }[\alpha, T] \text {, }
$$

i.e., the sequences $\left\{x_{n_{k}}\right\}$ and $\left\{x_{n_{k}}^{\prime}\right\}$ converge uniformly on the interval $[\alpha, T]$ to $x_{\alpha}$ and $x_{\alpha}^{\prime}$, respectively. Obviously, (4.7) and (4.8) are valid in particular for the elements of $\left\{x_{n_{k}}\right\}$ and $\left\{x_{n_{k}}^{\prime}\right\}$, respectively, from which, letting $k \rightarrow \infty$, one finds

$$
\begin{aligned}
& A_{1} \leq x_{\alpha}(t) \leq \tilde{M}_{0} \quad \text { for } t \in[\alpha, T] \\
& m_{1} \leq x_{\alpha}^{\prime}(t) \leq \phi_{\alpha}(\alpha)<B \quad \text { for } t \in[\alpha, T]
\end{aligned}
$$

Clearly, the functions $x_{n_{k}}(t), k \in \mathbb{N}, n_{k} \geq n_{\alpha, \mu}$, satisfy integral equations of the form

$$
x_{n_{k}}^{\prime}(t)=x_{n_{k}}^{\prime}(\alpha)+\int_{\alpha}^{t} f\left(s, x_{n_{k}}(s), x_{n_{k}}^{\prime}(s)\right) d s, \quad t \in(\alpha, T] .
$$

Now, since $f(t, x, p)$ is uniformly continuous on the compact set $[\alpha, T] \times\left[A_{1}, \tilde{M}_{0}\right] \times$ $\left[m_{1}, \phi_{\alpha}(\alpha)\right]$, from the uniform convergence of $\left\{x_{n_{k}}\right\}$ it follows that the sequence $\left\{f\left(s, x_{n_{k}}(s)\right.\right.$, $\left.\left.x_{n_{k}}^{\prime}(s)\right)\right\}, n_{k} \geq n_{\alpha, \mu}$ is uniformly convergent on $[\alpha, T]$ to the function $f\left(s, x_{\alpha}(s), x_{\alpha}^{\prime}(s)\right)$, which means

$$
\lim _{k \rightarrow \infty} \int_{\alpha}^{t} f\left(s, x_{n_{k}}(s), x_{n_{k}}^{\prime}(s)\right) d s=\int_{\alpha}^{t} f\left(s, x_{\alpha}(s), x_{\alpha}^{\prime}(s)\right) d s
$$


for each $t \in(\alpha, T]$. Returning to the integral equation and letting $k \rightarrow \infty$ yield

$$
x_{\alpha}^{\prime}(t)=x_{\alpha}^{\prime}(\alpha)+\int_{\alpha}^{t} f\left(s, x_{\alpha}(s), x_{\alpha}^{\prime}(s)\right) d s, \quad t \in(\alpha, T],
$$

which implies that $x_{\alpha}(t)$ is a $C^{2}(\alpha, T]$-solution to the differential equation $x^{\prime \prime}=f\left(t, x, x^{\prime}\right)$ on $(\alpha, T]$. Besides, (4.6) implies

$$
m_{1} t+A \leq x_{\alpha}(t) \leq B t+A \quad \text { for } t \in[\alpha, T] .
$$

Further, we observe that if the condition $\left(\mathrm{S}_{2}\right)$ holds for some $\alpha>0$, then it is true also for an arbitrary $\alpha_{0} \in(0, \alpha)$. We will use this fact considering a sequence $\left\{\alpha_{i}\right\} \subset(0, \alpha), i \in \mathbb{N}$, with the properties

$$
\alpha_{i+1}<\alpha_{i} \quad \text { for } i \in \mathbb{N} \text { and } \lim _{i \rightarrow \infty} \alpha_{i}=0
$$

For each $i \in \mathbb{N}$ we consider sequences

$$
\left\{x_{i, n_{k}}\right\}, \quad n_{k} \geq n_{i+1, \mu}, k \in \mathbb{N}, n_{i+1, \mu}>\max \left\{\alpha_{i+1}^{-1},\left(B+k \alpha_{i+1}-\mu\right)^{-1}\right\}
$$

on the interval $\left[\alpha_{i+1}, T\right]$. Thus, we establish that each sequence $\left\{x_{i, n_{k}}\right\}$ has a subsequence $\left\{x_{i+1, n_{k}}\right\}, k \in \mathbb{N}, n_{k} \geq n_{i+1, \mu}$, converging uniformly on the interval $\left[\alpha_{i+1}, T\right]$ to any function $x_{\alpha_{i+1}}(t), t \in\left[\alpha_{i+1}, T\right]$, that is,

$$
\left\|x_{i+1, n_{k}}-x_{\alpha_{i+1}}\right\|_{1} \rightarrow 0 \quad \text { on }\left[\alpha_{i+1}, T\right]
$$

which is a $C^{2}\left(\alpha_{i+1}, T\right]$-solution to the differential equation $x^{\prime \prime}(t)=f\left(t, x(t), x^{\prime}(t)\right)$ on $\left(\alpha_{i+1}, T\right]$ and

$$
\begin{aligned}
& m_{1} t+A \leq x_{\alpha_{i+1}}(t) \leq B t+A \quad \text { for } t \in\left[\alpha_{i+1}, T\right] \\
& m_{1} \leq x_{\alpha_{i+1}}^{\prime}(t) \leq \phi_{\alpha}\left(\alpha_{i+1}\right)<B \quad \text { for } t \in\left[\alpha_{i+1}, T\right] \\
& x_{\alpha_{i+1}}(t)=x_{\alpha_{i}}(t) \text { and } x_{\alpha_{i+1}}^{\prime}(t)=x_{\alpha_{i}}^{\prime}(t) \text { for } t \in\left[\alpha_{i}, T\right] .
\end{aligned}
$$

The properties of the functions from $\left\{x_{\alpha_{i}}\right\}, i \in \mathbb{N}$, imply that there exists a function $x_{0}(t)$ which is a $C^{2}(0, T]$-solution to the equation $x^{\prime \prime}=f\left(t, x, x^{\prime}\right)$ on the interval $(0, T]$ and is such that

$$
m_{1} t+A \leq x_{0}(t) \leq B t+A \quad \text { for } t \in(0, T]
$$

hence $\lim _{t \rightarrow 0^{+}} x_{0}(t)=A$,

$$
\begin{aligned}
& m_{1} \leq x_{0}^{\prime}(t) \leq \phi_{\alpha}(t)<B \quad \text { for } t \in(0, T], \\
& x_{0}(t)=x_{\alpha_{i}}(t) \text { for } t \in\left[\alpha_{i}, T\right] \text { and } i \in \mathbb{N}, \\
& x_{0}^{\prime}(t)=x_{\alpha_{i}}^{\prime}(t) \quad \text { for } t \in\left[\alpha_{i}, T\right] \text { and } i \in \mathbb{N} .
\end{aligned}
$$


We have to show also that

$$
\lim _{t \rightarrow 0^{+}} x_{0}^{\prime}(t)=B
$$

Reasoning by contradiction, assume that there exists a sufficiently small $\varepsilon>0$ such that for every $\delta>0$ there is a $t \in(0, \delta)$ such that

$$
x_{0}^{\prime}(t)<B-\varepsilon .
$$

In other words, assume that for every sequence $\left\{\delta_{j}\right\} \subset(0, T], j \in \mathbb{N}$, with $\lim _{j \rightarrow \infty} \delta_{j}=0$, there exists a sequence $\left\{t_{j}\right\}$ having the properties $t_{j} \in\left(0, \delta_{j}\right), \lim _{j \rightarrow \infty} t_{j}=0$ and

$$
x_{0}^{\prime}\left(t_{j}\right)<B-\varepsilon .
$$

It is clear that every interval $\left(0, \delta_{j}\right), j \in \mathbb{N}$, contains a subsequence of $\left\{t_{j}\right\}$ converging to 0 . Besides, from (4.9) and (4.10) it follows that for every $j \in \mathbb{N}$ there are $i_{j}, n_{j} \in \mathbb{N}$ such that $\alpha_{i_{j}}<\delta_{j}$ and

$$
\left\|x_{i, n_{k}}^{\prime}-x_{0}^{\prime}\right\| \rightarrow 0 \quad \text { on }\left[\alpha_{i}, \delta_{j}\right)
$$

for all $i>i_{j}$ and all $n_{k} \geq \max \left\{n_{i, \mu}, n_{j}\right\}$. Moreover, since the accumulation point of $\left\{t_{j}\right\}$ is 0 , for each sufficiently large $j \in \mathbb{N}$ there is a $t_{j} \in\left[\alpha_{i}, \delta_{j}\right)$ where $i>i_{j}$. In summary, for every sufficiently large $j \in \mathbb{N}$, that is, for every sufficiently small $\delta_{j}>0$, there are $i_{j}, n_{j} \in \mathbb{N}$ such that for all $i>i_{j}$ and $n_{k} \geq \max \left\{n_{i, \mu}, n_{j}\right\}$ from (4.12) and (4.13) we have

$$
x_{i, n_{k}}^{\prime}\left(t_{j}\right)<B-\varepsilon,
$$

which contradicts to the fact that $x_{i, n_{k}}^{\prime}(0)=B-n_{k}^{-1}$ and $x_{i, n_{k}}^{\prime} \in C[0, T]$. This contradiction proves that (4.11) is true.

Now, it is easy to verify that the function

$$
x(t)= \begin{cases}A, & t=0, \\ x_{0}(t), & t \in(0, T],\end{cases}
$$

is a $C[0, T] \cap C^{2}(0, T]$-solution to (1.1), (1.2). This function is strictly increasing because $x^{\prime}(t)=x_{0}^{\prime}(t) \geq m_{1}>0$ for $t \in(0, T]$, and the bounds for $x(t)$ and $x^{\prime}(t)$ follows immediately from the corresponding bounds for $x_{0}(t)$ and $x_{0}^{\prime}(t)$.

The following results provide information about the presence of other useful properties of the assured solutions. Their correctness follows directly from Theorem 4.2.

Theorem 4.3 Let $A \geq 0$ and let $\left(\mathrm{S}_{1}\right)$ and $\left(\mathrm{S}_{2}\right)$ hold. Then the singular IVP (1.1), (1.2) has at least one strictly increasing solution in $C[0, T] \cap C^{2}(0, T]$ with positive values for $t \in(0, T]$.

\section{Examples}

Example 5.1 Consider the IVP

$$
\begin{aligned}
& x^{\prime \prime}=\frac{\sqrt{b^{2}-x^{2}}}{\sqrt{c^{2}-t^{2}}} P_{k}\left(x^{\prime}\right), \\
& x(0)=0, \quad x^{\prime}(0)=B, \quad B>0,
\end{aligned}
$$


where $b, c \in(0, \infty)$, and the polynomial $P_{k}(p), k \geq 2$, has simple zeroes $p_{1}$ and $p_{2}$ such that

$$
0<p_{1}<B<p_{2}
$$

Let us note that here $D_{t}=(-c, c), D_{x}=[-b, b]$ and $D_{p}=\mathbb{R}$.

Clearly, there is a sufficiently small $\theta>0$ such that

$$
0<p_{1}-\theta, \quad p_{1}+\theta \leq B \leq p_{2}-\theta
$$

and $P_{k}(p) \neq 0$ for $p \in\left[p_{1}-\theta, p_{1}\right) \cup\left(p_{1}, p_{1}+\theta\right] \cup\left[p_{2}-\theta, p_{2}\right) \cup\left(p_{2}, p_{2}+\theta\right]$.

We will show that all assumptions of Theorem 3.2 are fulfilled in the case

$$
P_{k}(p)>0 \quad \text { for } p \in\left[p_{1}-\theta, p_{1}\right) \quad \text { and } \quad P_{k}(p)<0 \quad \text { for } p \in\left(p_{2}, p_{2}+\theta\right] \text {; }
$$

the other cases as regards the sign of $P_{k}(p)$ around $p_{1}$ and $p_{2}$ may be treated similarly. For this case choose $\tau=\theta / 2, m_{1}=p_{1}>0$ and $M_{1}=p_{2}$. Next, using the requirement $\left[A-\tau, M_{0}+\right.$ $\tau] \subseteq[-b, b]$, i.e. $\left[-\theta / 2, p_{2} T_{0}+\theta / 2\right] \subseteq[-b, b]$, we get the following conditions for $\theta$ and $T$ :

$$
-\theta / 2 \geq-b \text { and } p_{2} T_{0}+\theta / 2 \leq b
$$

which yield $\theta \in(0,2 b]$ and $T \leq \frac{2 b-\theta}{2 p_{2}}$. Besides, $[0, T] \subseteq(-c, c)$ yields $T<C$. Thus, $0<T<$ $\min \left\{c, \frac{2 b-\theta}{2 p_{2}}\right\}$. Now, choosing

$$
\bar{m}_{1}=p_{1}-\theta \text { and } \quad \bar{M}_{1}=p_{2}+\theta
$$

we really can apply Theorem 3.2 to conclude that the considered problem has a strictly increasing solution $x \in C^{2}[0, T]$ with $x(t)>0$ on $t \in(0, T]$ for each $T<\min \left\{c, \frac{2 b-\theta}{2 p_{2}}\right\}$.

Example 5.2 Consider the IVP

$$
\begin{aligned}
& x^{\prime \prime}=\frac{\left(x^{\prime}-5\right)\left(15-x^{\prime}\right)}{(x-2)^{2}\left(x^{\prime}-10\right)}, \\
& x(0)=2, \quad \lim _{t \rightarrow 0^{+}} x^{\prime}(t)=10 .
\end{aligned}
$$

Notice that here

$$
S_{A}=\mathbb{R} \times\{2\} \times((-\infty, 10) \cup(10, \infty)), \quad S_{B}=\mathbb{R} \times((-\infty, 2) \cup(2, \infty)) \times\{10\} .
$$

It is easy to check that $\left(\mathrm{S}_{1}\right)$ holds, for example, for $\bar{m}_{1}=4, m_{1}=5, v=0.1$, and an arbitrary fixed $T>0$, moreover, $\tilde{M}_{0}=10 T+3$. Besides, for $k=-24 /(10 T+1)^{2}, \alpha=T / 100$ and $\mu=9$, for example, we have

$$
k \alpha+B=-24 T / 100(10 T+1)^{2}+10>9=\mu
$$

and $f(t, x, p) \leq-24 /(10 T+1)^{2}$ on $[0, T / 100] \times(2,10 T+3] \times[9,10)$, which means that $\left(\mathrm{S}_{2}\right)$ also holds. By Theorem 4.3, the considered IVP has at least one positive strictly increasing solution in $C[0, T] \cap C^{2}(0, T]$. 


\section{Competing interests}

The authors declare that they have no competing interests.

\section{Authors' contributions}

All authors read and approved the final manuscript.

\section{Author details}

${ }^{1}$ Department of Mathematics, Technical University of Sofia, Branch Sliven, Sliven, Bulgaria. ${ }^{2}$ Faculty of Mathematics, 'St. Kl. Ohridski' University of Sofia, Sofia, Bulgaria.

\section{Acknowledgements}

The work is partially supported by the Sofia University Grant 158/2013 and by the Bulgarian NSF under Grant DCVP 02/1/2009.

\section{Received: 30 January 2014 Accepted: 13 June 2014 Published online: 26 September 2014}

\section{References}

1. Rachůnková, I, Tomeček, J: Bubble-type solutions of non-linear singular problem. Math. Comput. Model. 51, 658-669 (2010)

2. Rachůnková, I, Tomeček, J: Homoclinic solutions of singular nonautonomous second-order differential equations. Bound. Value Probl. 2009, Article ID 959636 (2009)

3. Rachůnková, I, Tomeček, J: Strictly increasing solutions of a nonlinear singular differential equation arising in hydrodynamics. Nonlinear Anal. 72, 2114-2118 (2010)

4. Agarwal, RP, O'Regan, D: Second-order initial value problems of singular type. J. Math. Anal. Appl. 229, 441-451 (1999)

5. Yang, G: Minimal positive solutions to some singular second-order differential equations. J. Math. Anal. Appl. 266, 479-491 (2002)

6. Yang, G: Positive solutions of some second-order nonlinear singular differential equations. Comput. Math. Appl. 45, 605-614 (2003)

7. Bobisud, LE, O'Regan, D: Existence of solutions to some singular initial value problems. J. Math. Anal. Appl. 133, 215-230 (1988)

8. Bobisud, LE, Lee, YS: Existence of monotone or positive solutions of singular second-order sublinear differential equations. J. Math. Anal. Appl. 159, 449-468 (1991)

9. Cabada, A, Heikkilä, S: Extremality results for discontinuous explicit and implicit diffusion problems. J. Comput. Appl. Math. 143, 69-80 (2002)

10. Cabada, A, Cid, JA, Pouso, RL: Positive solutions for a class of singular differential equations arising in diffusion processes. Dyn. Contin. Discrete Impuls. Syst., Ser. A Math. Anal. 12, 329-342 (2005)

11. Cabada, A, Nieto, JJ, Pouso, RL: Approximate solutions to a new class of nonlinear diffusion problems. J. Comput. Appl. Math. 108, 219-231 (1999)

12. Cid, JA: Extremal positive solutions for a class of singular and discontinuous second order problems. Nonlinear Anal. 51, 1055-1072 (2002)

13. Maagli, H, Masmoudi, S: Existence theorem of nonlinear singular boundary value problem. Nonlinear Anal. 46 465-473 (2001)

14. Zhao, Z: Positive solutions of nonlinear second order ordinary differential equations. Proc. Am. Math. Soc. 121, 465-469 (1994)

15. Kelevedjiev, P, Popivanov, N: On the solvability of a second-order initial value problem. Paper presented at the 40th international conference on the applications of mathematics in engineering and economics, Technical University of Sofia, Sozopol, 8-13 June 2014

16. Granas, A, Guenther, RB, Lee, JW: Nonlinear boundary value problems for ordinary differential equations. Diss. Math. 244, 1-128 (1985)

17. Grammatikopoulos, MK, Kelevedjiev, PS, Popivanov, N: On the solvability of a singular boundary-value problem for the equation $f\left(t, x, x^{\prime}, x^{\prime \prime}\right)=0$. J. Math. Sci. 149, 1504-1516 (2008)

18. Kelevedjiev, P, Popivanov, N: Second order boundary value problems with nonlinear two-point boundary conditions. Georgian Math. J. 7, 677-688 (2000)

19. Palamides, P, Kelevedjiev, P, Popivanov, N: On the solvability of a Neumann boundary value problem for the differential equation $f\left(t, x, x^{\prime}, x^{\prime \prime}\right)=0$. Bound. Value Probl. 2012, 77 (2012). doi:10.1186/1687-2770-2012-77

20. Kelevedjiev, P: Positive solutions of nonsingular and singular second order initial value problems. Int. Electron. J. Pure Appl. Math. 2, 117-127 (2010)

doi:10.1186/s13661-014-0161-z

Cite this article as: Kelevedjiev and Popivanov: Second-order initial value problems with singularities. Boundary Value Problems 2014 2014:161. 